\title{
An Analysis of the Writing Problems of Ghanaian ESL Students: A Focus on Textual Dimension
}

\author{
Tabiri Francis \\ Department of Arts Education, University of Cape Coast, Cape Coast, Ghana
}

\begin{abstract}
Reading becomes interesting and enjoyable when texts are organized or structured in relation to progression in the ordering of utterance themes and their rhemes. It appears, therefore, that ESL students have problems with this aspect of writing as the intricacies and complexities of writing have been noted (Adas \& Bakir, 2013), but previous studies seem to focus largely on the problems below the clause level. Therefore, focusing on 15 compositions written by form three students in the Sunyani Senior High School, the study examines ESL students' problems in the organization of lexico-grammatical resources in text creation. Results show that ESL students face problem of proper thematic progression, which results in disunity in the development of a paragraph and incoherent sentences within paragraphs. This comes as a result of the introduction of a brand new theme and a misplaced theme. The study also shows, in terms of proper structuring of sentences that ESL students' sentences are rather in fragment, run-on and phrases. It is suggested that teachers of English language should sensitize students to read extensively and engage students in a lot of practical exercises in writing in order to help students to improve upon their writing skills.
\end{abstract}

Keywords: Essay, Writing Problems, Thematic Progression, Rheme

DOI: $10.7176 / \mathrm{RHSS} / 9-20-02$

Publication date:October $31^{\text {st }} 2019$

\section{Introduction}

English language gained its root in Ghana in the $16^{\text {th }}$ century through the initial contact with British. English emerged as the language of trade, education, governance, and as cross-ethnic lingua franca (Sey, 1973). The colonial and missionary language policy saw to the consolidation of English language in the country. Of significance is the role of Reverend Denny who became school master of the Cape Coast castle in 1824 . He advocated the exclusive use of English language in the school, violations of which attracted a penalty. Further, the missionaries also saw the use of English as vital in their missionary work and therefore English was used in several Wesleyan mission schools.

From its first implantation in Ghana, then the Gold Coast, in the early part of the $16^{\text {th }}$ century to date, English language in Ghana, like in other West African countries has shown formidable resilience as the language of formal education, and a medium for cross-ethnic communication in a predominantly multilingual environment (Adika 2012). Whatever the subject, whatever the area of specialisation, we need to acquire proficiency in English language to function. When we examine the goals of English language teaching in Ghana, it is clear that English is taught to enable us to use the language to communicate effectively. We need English to pursue studies in other subjects, for further studies, employment, social, recreational and administrative purposes (Amua-Sekyi, 2010). Thus, English language is needless to say, the language of instruction in Ghanaian Schools, colleges and universities. It is also the language of all formal professions in Ghana. In Ghanaian schools, all subjects such as Mathematics, science, social studies and others are taught and learned through the use of English language. English language usage as a matter of fact has become imperative in Ghana today.

Clearly, the role of English language in the social, economic, political and most importantly, educational lives of Ghanaians cannot be overemphasised. Of particular essence is writing. Thus, it can be said that the majority of tasks, most especially academic tasks, in most parts of the country are accomplished by different forms of writing in the English language. English language, in our contemporary lives, makes a strong contribution to education, for example, texts books, hand-outs, examination and others are all enacted through the use of English language. Notwithstanding, in Ghana, where English language is considered as lingua franca and official language for interethnic communication among the country's several ethno-linguistic groups (Afful, 2007), the most serious problem in English language learning is writing skills (Chief Examiner's report, 2018). According to the report, some students could not write full length essay. Amua-Sekyi (2010) has also provided evidence of the illogical construction on the part of Ghanaian students. It is therefore crucial to expose Ghanaian students to the depth of errors descendible in their written essays, and the reason for the occurrence of such errors. With regard to the teaching of English language as a second language in Ghana, many studies have been done to ascertain errors which occur below the clause level (mechanics of the language), but there are few works that focus on errors at the discourse level. This lacuna in previous studies therefore gives me the impetus to conduct a study of Ghanaian learners' writing to ascertain their problems of the organisation of lexico-grammatical resources in texts creation. This study is guided by the following research questions. 
1. What problems are discernible in Ghanaian students' written essays in respect of the thematic progression?

2. What problems are identifiable in Ghanaian students' written essays in terms of sentence construction?

\subsection{Theoretical framework}

\subsection{Danes' Theoretical Framework of Thematic Progression - Textual meta-function}

Danes (1974) points out that the organization of information in texts is determined by the progression in the ordering of utterance themes and their rhemes. Danes' view on the relationship between successive themes and their rhmes would appear to provide a more satisfactory account of the 'method of development' of texts. Thus, Danes makes two revealing aspects of the property of being New: first, New may mean something not mentioned before in the preceding context, and also, New may mean something related as rheme to a theme to which it has not yet been related. The thematic organisation of the text is closely related to discourse coherence or text connectivity. A text is defined as text largely in terms of its semantic coherence, but Danes indicates that texts are not always perfect; they do not only display coherence to an uneven degree, but some may be characterized as 'discontinuous'. The observance of coherence as a rule in paragraph is probably one of the causes of difficulty in the teaching of academic writing.

Textual meta-function is realised through thematic structure, information structure and cohesion. Thematic structure includes theme and thematic progression pattern, information structure includes given and new units of information as espoused by Danes (1974); cohesion includes reference, conjunction, ellipsis as well as lexical cohesion. Hence, to be able to organize a text into a coherent whole, writers and speakers need to keep their readers and listeners well informed about where they are going and should stay focus on that direction (Arancon, 2013; Arunsirot, 2013)

Theme is the first element in the clause, which functions as the starting point or signpost to signal what the message is about (Butt et al. cited in Arancon, 2013). According to Halliday (as cited in Arancon, 2013), theme is "what the message is concerned with: the point of departure for what the speaker is going to say or the element the speaker selects for grounding what he is going to say". The rest of the clause is called the rheme, which is the point of the clause where the meanings have been heading (Butt et al. cited in Arancon, 2013). He advances that theme is Given or what is already known or accessible to the reader, and New is that which generates information in a clause. Themes can be divided into three main types: topical, textual and interpersonal. Topical theme foregrounds experiential meaning. Textual theme highlights relationships between parts of text. Interpersonal theme is shown through the use of finite and modal elements. The ideational themes can further be divided into unmarked and marked. Unmarked themes are grammatical sentence subject in declarative clauses and can be realized as simple and complex nominal groups. The marked themes can be further categorized as: circumstantial adjunct, subordinating clause and attributive clause (Arancon, 2013; Arunsirot, 2013). Examples as taken from ESL students' essays to further explain the concept of theme and rheme are presented in Table 1.

Table 1: Examples of Theme and Rheme

\begin{tabular}{|c|c|c|c|c|}
\hline \multicolumn{4}{|c|}{ Theme } & Rheme \\
\hline \multirow[t]{2}{*}{ Textual theme } & Interpersonal theme & \multicolumn{2}{|l|}{ Topical theme } & \\
\hline & & marked & unmarked & \\
\hline \multirow[t]{2}{*}{$\begin{array}{l}\text { First, second, } \\
\text { then, because etc. }\end{array}$} & $\begin{array}{l}\text { Most especially, I } \\
\text { think, I believe etc. }\end{array}$ & \multirow[t]{2}{*}{$\begin{array}{l}\text { In the meantime, if } \\
\text { you come etc }\end{array}$} & & Is a very simple dish. \\
\hline & & & They & $\begin{array}{l}\text { Taught us to better } \\
\text { understand. }\end{array}$ \\
\hline
\end{tabular}

\subsection{Previous Studies on Students' Writing Skills}

Klu (2014) admits that students are not proficient in the use of grammatical concord. That is, he focuses on an analysis of grammatical concord in some selected examination scripts of students of the Ghana Technology University College. Thus, dwelling on a purposeful critical discourse analysis in analysing sentences from selected scripts of level 200 students in order to ascertain their proficiency in the use of grammatical concord in English, it becomes very clear that the students struggle mostly with the concord within tenses. They also seem to have problem with concord with relative pronouns and the subject verb agreement, particularly with pseudoconjunctions such as: as well as, in addition to, together with etc. Omotese (2012) also focuses on a comparison of errors of concord students often conflict when speaking or writing essays, and identifies an increase in errors resulting to $3.40 \%$ among students in the first year senior secondary but with drastic reduction of $10.48 \%$ among the third year students of senior secondary school which according to him, performed below expectation. Several studies have been conducted by researchers on different aspects of concord errors: problems regarding irregular or unmarked plural nouns, problem with respect to $3^{\text {rd }}$ person pronoun and noun phrases as subjects, the problem regarding the long distance between the subject and the predicate as well as the problem with collective nouns (Thagg-Fisher, Köhlmyr, Levin cited in Omotese, 2012).

In line with writing problems of students below the clause level, and by focusing on the test item of 
unpunctuated passage as an instrument, Akampirige and Apam (2014) have revealed that an overwhelming majority $(63 \%)$ of the students drawn for the study cannot differentiate between the colon as well as the semicolon. This result is replicated by Msanjila (2005) who identifies the problem with the use of the semi colon and the colon among Kiswahili students in Tanzanian. Ghabool, Mariadass and Kashef (2012) equally explore the writing problems of students and identify punctuation to be a major problem for students. Other areas with respect to students' writing problems below the clause level explored by researchers include: the use of determiners, tenses, prepositions, adverbial and adverbs, adjectives and pronouns (Lawoyi and Adeyanju, 2013); spelling problems of students (Krishnamurthy, Kangira, Tjiramanga \& Beukes, 2010; Adas \& Bakir, 2013).

Msanjila (2005), however, focuses on essay test in analysing the writing problems of Kiswahili students in Tanzania. By focusing on a small number of students, the study identifies that the students have problems with capitalisation and punctuation, inexplicitness or fuzziness, poor organization or illogical sequence, spelling and grammar. The study further reveals that the misuse of capital letters is the common problem that characterises the writing of the students, which ranks high among all the problems identified in the students' writings. Amua-Sekyi (2010), like Msanjila (2005), focusing on document analysis, identifies that Ghanaian university students struggle with the syntactic aspect of their language. According to her, many students struggle to express themselves, produce concepts that are vague and in a language that is inadequate for their needs; the results are logically incoherent discourse that is commonly produced under examination conditions.

Quite clearly, previous works on students' writing problems as realised from the foregoing, reveal a considerable attention given to the problems below the clause level. It is for this reason that the current study focuses on students' writing problems at the clausal level from the perspective of Dane's Thematic Progression.

\subsection{Methodology}

This is a qualitative study, which allows an in-depth description, analysis and interpretation of a text (Snape \& Spencer, 2003; Ogah, 2013). The study employs the documentation method. This is to allow for a comprehensive analysis of the students' writing skills in their essays. In all, 15 compositions written by form three students of Sunyani Senior High School were used, particularly 2015-2016 academic year. These students were in their final year and were ready to write their final exams. The choice of the final year students was informed by the fact that these students have had enough writing practices as they have gone through most of the pertinent issues regarding writing. For this reason, the essay topic was directly taken from English language past questions of the West Africa Secondary School Certificate Examinations. They were asked to select one essay question from different topics and write full essay as required from them in their final examination. The questions are given below:

1. There has been an outbreak of an epidemic in your area. Write a letter to the Editor of the national newspaper discussing the causes and effects of this problem (SSCE June, 1995).

2. You have changed school and after a few months, you discover that your present school is not as good as the former. Write a letter to your father giving at least three areas in which the new school does not measure up to the old one (SSCE June, 2003).

3. One of your pen friends has written asking you to describe your favourite Ghanaian dish to enable her to prepare it. Write back to her explaining how the dish is cooked (WASSE past question, 1998).

Given that the study sought to investigate the students' problems in writing English, two aspects of the organisation of lexico-grammatical resources in texts creation were looked at. These were the "thematic progression" and "sentence construction." This analysis of the choice of Theme and Rheme allows the revelation of the writing problems with topic development and text flow that Ghanaian ESL learners' experience.

\subsection{Analysis and discussion}

This paper dwelled on exploring the problems that are discernible in students' written essays from the perspective of textual meta-function. The analysis and discussions are given below.

\subsection{Organisation of Lexico-grammatical Resources in Texts Creation}

This research question dwelled on the kind of problems ESL students face when putting their information together to form a text. Danes' thematic structure, information structure and cohesion in the organisation of a text were the focus of the analysis of this research question. A thorough examination of the students' essays revealed that ESL students struggle with thematic progression which results in improper development of information in paragraphs and illogical sequence of ideas in sentences within a paragraph. These problems are discussed below.

\subsubsection{The Problem of Improper Thematic progression}

Thematic progression refers to the situation where the theme of the clause may pick up or repeat a meaning from a preceding theme or rheme to help achieve cohesion or unity and coherence in a text, which helps readers in a logical and rational course (Paltridge cited in Arunsirot, 2013). However, analysis of ESL students' essays revealed the addition of a brand new theme and misplaced theme which results in disunity and incoherent sentences in their paragraphs. 


\subsubsection{The Problem of Disunity or Brand New Theme}

Analysis of ESL students' essays also revealed that students introduce brand new themes in their thematic progression, resulting in disunity of sentences in their paragraphs. This is what Msanjila (2005) refers to as poor organisation of paragraphs. A paragraph contains one central idea, such that all the sentences, ideas and images are controlled by the single purpose of focus (same theme). However, ESL students bring different ideas or new themes, which should be developed in different paragraphs into one paragraph; thereby resulting in disunity. Also, instead of the students treating a single idea (same theme) in each paragraph in order to achieve unity, they split the idea into separate paragraphs. Below are some extracts taken from students' essay to further explain this problem.

\section{Example 10}

a. Nevertheless, some people contract this disease at their own fault, people who practice unhygienic life is at high risk of getting the so called "devil vomit". People who use their fingers to remove concentrated dirt from their nose and forget to wash their hands before eating is likely to contract this disease.

In addition to this, some of us who fail to wash our bowls after eating and before eating are at high risk of contracting cholera (PC 62).

b. I am very grateful to write you this letter, by the way, how is your health condition. I know by the Almighty father you are leaving an excellent health as am I here.

The reason why I am writing this letter is about at least three areas in which the new school does not measure up to the old one. Daddy, to go straight to my points, Firstly my formal is a school of with great ambitions and there is a saying ambition is the key to development. My formal school has good infrastructural building and learn materials. From this point, my new school has bad infrastructural building as to this result, there are not even teachers... (PC 65).

c. My favourite Ghanaian dish is yam with kontomire stew. You first peal the yam and put them in a cooking pot containing water but first clean them well with a small amount of salt. After that you boil it completely. After you drawn the water from it and deep it covered to prevent heat loss. Then boil the kontomire for a few min to avoid loss of energy with vegetables that will be used for the stew which are; pepper, tomato, onion. After boiling, grind the pepper with the onion and then add the tomato and grind well till you see no seeds... (PC 61).

As could be seen in the extracts above, ESL students lack clear sense of paragraphing and so they either merge two different controlling ideas (introducing a brand new theme) in one paragraph or dissect an idea into separate paragraphs. In example $10 a$, the student's controlling idea is that people's own practice which is personal hygiene brings about cholera, which is the theme in the topic sentence. As part of the paragraph, and in developing the issue of personal hygiene, the student talks about unwashed hands before eating, which is another theme in the first major support sentence. Equally, not washing one's bowls as he talks about is another aspect of personal hygiene; another theme in the second major support sentence and therefore should be brought together in one paragraph. However, instead of the student to talk about the habit of not washing one's bowls after cooking as an issue of personal hygiene to make it one idea so as to help develop the controlling idea, the student rather captures it in another paragraph.

Similarly, the extract in example 10b depicts ESL students' difficulties in thematic progression in paragraph development. Thus, the first sentence in the student's second paragraph should have been captured as part of the first paragraph, which is the introductory paragraph. Clearly, that can be taken as the thesis statement, but the student stated it as part of the main body. Quite apart from that, the student's second sentence in the second paragraph focuses on ambition (Theme), whereas the third sentence in the same paragraph looks at infrastructural development (brand new Theme). This indicates that the student is raising two different points or themes making different ideas, which should therefore be put in separate paragraphs.

Example 10c is no exception so far as this problem is concerned as it seeks to substantiate ESL students' problems with unity in paragraphing. As evident in the extract in example 10c, the student is committed to describing a favourite Ghanaian food, which is taken to be yam (brand new theme) and palaver sauce stew (brand new theme) as he refers to as kontomire; and in a bid to do that, provides all the information about these new themes in a single paragraph. Meanwhile, the student could have separated the preparation of the yam from that of the stew. This exemplifies the kind of challenge they face in respect of putting their ideas in paragraphs.

\subsubsection{The Problem of a Misplaced Theme}

Proper progression of theme ensures that sentences within and beyond paragraphs are presented or structured such that all the sentences are orderly and systematically arranged to achieve coherence. This is the orderly or logical arrangement of sentences in a paragraph. ESL students, however, provide misplaced themes, which result in incoherent sentences within a paragraph. Coherence is therefore the orderly or logical arrangement of sentences and ideas in a paragraph. Msanjila (2005) captures this as inexplicitness or fuzziness. Analysis of the students' essays shows that Ghanaian ESL students have difficulty structuring their ideas in a logical manner. This order of presentation makes it difficult for readers to be able to follow the terrain of thoughts of the writer and get vividly 
the relevant mental picture of what the writer is talking about, which results in inexplicitness or fuzziness as Msanjila talks about. Let us consider the examples below.

\section{Example 11}

a. Secondly, lack of good drinking water and bad sanitation. Daddy this point in my major point and due to that there is outbreak of an epidermic disease, in which my health is also a concern father to you and I. Due to that, our school children take in the contaminated water and results in typhoid fever which is also a deadly disease. Comparing to my formal school, this problem even makes me afraid in my present school and does not suit me daddy. Bad sanitation is also a factor which the new school does not measure up to the old one (PC65).

The above paragraph has unity - its focus being lack of good drinking water and sanitation; but it lacks coherence (misplaced theme). An aspect of the student's dislike for the effect the situation has on students in the school is put between a discourse on the events leading to the epidemic, and the student's dislike is continued elsewhere. Also, logically, taking in contaminated water may result in typhoid fever which may lead to the epidemic. However, the student mentions the issue of epidemic and talks about his health before talking about the issue of taking in contaminated water. Again, the use of the connector due to that in the second sentence as a transition to the issues regarding the epidemic is misplaced, as it does not connect directly to the preceding rheme. Therefore, the connector due to that lacks logical sequence. In like manner, the link between the second sentence, and the third sentence: ... in which my health is also a concern father to you and I and our school children take in the contaminated water... by the use of due to that is a misplaced theme and therefore, lacks logical connection; thereby making the construction above nebulous. That is, there is no clear connection between the second sentence and the third sentence. Equally, the paragraph below throws more light on students' illogical sequence of ideas:

b. First and foremost, overcrowded settlement is one of the causes of an epidermic in our area. House are closely packed in our area. So when there is any disease like chicken pox which is an air borne disease can be spread easily. Also, overcrowded settlement does not get air to pass through the environment. A lot of heat in the environment can cause rashes to people in the environment (PC 5)

Much can be said about the paragraph quoted above. For instance, the third sentence of the first paragraph which starts with also does not explicitly mark the direct relationships with the preceding sentence (misplaced theme). The third sentence states something quite different from the first sentence. This implies that the element also which functions as a link device has not been used appropriately. There is therefore the difficulty in appreciating whether the sentence; Also, overcrowded settlement... directly follows: So when these is any disease like chicken pox which is an air borne disease can be spread easily or the latter rather directly follows the former; hence leaving readers to decipher the relationship and the intended meaning. Again, the student begins to develop an idea: Houses are closely... which seeks to explain the issue of overcrowding, but stops and introduce another issue: So when this is... and continues later somewhere in the paragraph. Thus, there is a back and forth reading which impedes the free flow of ideas. This therefore breaches coherence as a characteristic of a good paragraph. This result follows directly the findings of Msanjila (2005) on an account of the problems ESL students face when writing, as he identifies among Tanzania students the problem of inexplicitness or fuzziness to be a challenge. According to him, students do not seem to appreciate the value of explicitness in written communication. Relationships of elements within and across sentences are not clearly marked. The syntax is predominantly elliptical and markedly Para tactical. The reader is left to infer the intended relationships. He attributes this problem to bad teaching methodology. Similar finding is reported by Amua-Sekyi (2010) as she asserts that students find themselves struggling to express concepts. Their expression are vaguely understood; the results are logically incoherent discourse that is commonly produced under examination conditions.

\subsection{The Problem of Faulty Sentence Construction}

Another identifiable problem in ESL students' written essays is their inability to structure their sentences in a proper manner. ESL students' sentences are faulty. These faulty sentences dwell on sentence fragment and run on sentence. They also use phrases as if they were sentences.

\subsubsection{Sentence Fragment}

It can be identified in the students' essays that they present or punctuate a construction which is not a sentence as though it is. This problem exists because the students fail to recognise illustration phrases which must be included in the main sentence, comment clauses which are dependent and thus, must be included in the main clause and also take phrases for sentences (Sekyi-Baidoo, 2003), resulting in an undue segregation of themes. As Dane's framework of thematic progression has revealed, to be able to offset this problem, there must be a proper use of cohesive devices or connectors. According to Zamel cited in Alfaki (2015) cohesive devices are crucial in writing, and hence when used inappropriately, result in faulty sentences.

Example 12: illustration phrases

ESL students fail to appreciate that illustration phrases are part of sentences and therefore detach them from their 
sentences and make them stand on their own as in:

a. ... there has been a floor in my area which has destroyed many life and properties of the people in my area. Such as cars, houses and etc (PC 64).

b. ... wash all the ingredients needed for preparation. Cut tomatoes, onions, kontomire into suitable sizes, grind pepper and onion take from earthenware and grind agushi (PC 71).

In both examples $12 a$ and $b$, the students fail to recognize that Such as cars, houses and etc.; Cut tomatoes, onions, kontomire into suitable sizes, grind pepper and onion take from earthenware and grind agushi are phrases that provide illustrations to the sentences ... there has been a floor in my area which has destroyed many life and properties of the people in my area and ... wash all the ingredients needed for preparation, and therefore must be brought together. In example $a$, the textual theme "such as" has unduly been separated from the rheme "properties of my area." Hence, joining the textual theme to the rheme, the sentence can be corrected by simply taken away the full-stop as in: there has been a floor in my area which has destroyed many lives and properties of the people in my area such as cars, houses and others. The same thing can be done with example $b$.

\section{Example 13: Comment Clauses}

The anomaly of using comment clauses as sentences are typical examples noted in the essays. Comment clauses explain or exemplify textual theme in the textual meta-function of the theoretical framework, which ESL students separate from the rest of the clausal elements (rheme), thereby depicting ESL students' lack of organisational skill in writing. The excerpts below demonstrate this problem.

a. "I once went to one class, two students were using one chair. And even the chairs are hard" (PC 55).

b. "Also in terms of supply of books. This new school is the school who supply bad books to the students. Because they cannot even identify good books form bad ones" (PC 56).

In example $a$ above, the full-stop can be removed and replaced by a comma, semi-colon or dash so that the italicised portion, which is the fragment, will be joined to the main part of the sentence. In respect of example $b$, the first structure is not a sentence, and hence the full-stop can be taken away and allow it to be joined to the second structure by introducing a comma before the second structure; the capital $T$ in the second structure must be changed to a small $t$. Also, the third structure is a dependent clause and so cannot stand alone as a sentence; so the full-stop before it must be taken away so that it is joined to the main clause. In line with this faulty sentence construction, Alfaki (2015) identifies that students have problem with the use of linking devices and proper punctuations.

\section{Example 14: Phrases as Sentences}

Another problem that can be identified in the students' written essays is their inability to recognise that academic essays demand that all ideas must be presented in sentences, and that a sentence contains a subject and predicate. This means every sentence should make meaning or give a complete thought (Sekyi-Baidoo, 2003). That is, the students introduce their theme but without a rheme as is the case in a clause. This problem results in faulty constructions like:

a. Firstly, inadequate social amenities (PC 69).

b. Secondly, inadequate skilled and unskilled labour (PC 10).

c. Daddy, well trained teachers (PC 4).

d. To continue, bad attitudes towards teaching and learning (PC 68).

e. Firstly, academic excellence at the end of final year exams (PC 67).

A careful examination of the examples above reveals that the students are confused about the complex noun phrase and what qualifies as a sentence. Thus, examples $14 a$ to $e$ are all phrases since none of them can be said to be meaningful; hence cannot end with a full-stop as if they were sentences as is the case in the above extracts.

\subsubsection{Run on Sentences}

While some of the students put their sentences in separate units, thereby resulting in fragments, others fail to recognize that when independent clauses which could stay individually as sentences are put together as one sentence, there must be appropriate punctuation marks- comma, semi-colon, or conjunction; hence resulting in run-on-sentences. Kharma (as cited in Alfaki, 2015) has therefore identified that those students who have the problem of writing good sentence structures are unable to produce longer sentences requiring subordination and coordination. A run-on-sentence is somehow the opposite of a sentence-fragment. In one, there is an undue separation, causing fragmentation. In the other, there is undue integration which overflows normal boundaries (Sekyi-Baidoo, 2003, P. 500). Let us observe the following examples.

\section{Example 15: run on sentence}

a. Put sauce pan on fire and put in the sauce pan palm oil of reasonable quantity (1) when it is heated (2) put the salt fish in it, (3) add the cut onion leaf to fry a little (4) add grinded pepper (5) and also add the tomatoes (6) wait until it is well cooked (7) add a little salt to taste and put agushi in your stew leaf to cook (PC 71).

b. Firstly, you set up your fire and peel the yam (1) after peeling the yam (2) you wash it with water and then put it on a cooking pot (3) and put water on the yam and then you add salt to it (PC 42).

The examples above show a clear incident of run-on-sentences in ESL students' writings. Sekyi-Baidoo (2003) 
provides some path ways in which run-on sentences can be corrected. According to Sekyi-Baidoo, run-on sentences can be corrected by introducing both coordinating and subordinating conjunctions- and, but, so, because, for and others. It can also be corrected by the introduction of punctuation marks, and also, changing main clauses or sentences to phrases or subordinate clauses. In the examples above, numbers have been assigned to areas that should have seen punctuations or co-ordinations to show instances of run-on-sentences. In example $a$, the area marked 1 , should have received a full-stop or the coordinating conjunction and; the student should have placed comma at the area marked 2; with the area marked 3, the student should have put a colon or the coordinating conjunction and. At the place marked 5, the student should have placed a full-stop; there should be and at the portion marked 6 and finally full-stop or and should have been placed at number 7 . Hence, setting off the sentences that run on in this piece or extract without attention to any other grammatical errors, the sentence will read thus:

c. Put sauce pan on fire and put in the sauce pan palm oil of reasonable quantity. When it is heated, put the salt fish in it; add the cut onion leaf to fry a little and add grinded pepper. You then add the tomatoes and wait until it is well cooked and then add a little salt to taste and put "agushi" in your stew leaf to cook (PC 71). Similarly, the run on sentences in example $b$ can be corrected by introducing the appropriate markers, which will make the sentence read like:

d. Firstly, you set up your fire and peel the yam. After peeling the yam, you wash it with water and then put it on a cooking pot; and pour water on the yam and add salt to it (PC 42).

Clearly, these run-on-sentences as exemplified in the extracts above make reading difficult. For this reason, SekyiBaidoo (2003) asserts that run-on sentences create undue complexity. According to him, run-on sentences make reading as a whole very difficult and also bring about ambiguity. Similarly, Al Seyabi and Tuzlukova (2014) who when focusing on quantitative analysis on the writing problems among Omani students, identify that the students' major problem has to do with putting their ideas together in a coherent way, choosing right vocabulary to express ideas and expressing ideas about the suggested topic. Abdulkareem (2014) on the writing problems among Arab students, finds out that ESL students are unable to recognize sentences to be more effective academically, and that they commit many mistakes related to sentence structure. Msanjila (2005) with a similar finding among Kiswahili students in Tanzania on the problems they face in writing an academic essay, draw the source of this problem from students lack of extensive reading, and offers that the problem could be mitigated by the creation of a wider reading and writing habit. In line with the findings of this study, Tsegaye (as cited in Alfaki, 2015) finds out that students use run-on, incorrect, and fragmented sentences.

\subsection{Discussion and Conclusion}

Writing in a second or foreign language seems to be the most difficult language skill for language learners to acquire in academic contexts (Negari, 2011). Ghana is no exception when discussing students' writing difficulties of which several researchers have focused on over the years by dwelling particularly on the difficulties of students' writings below the clausal level. Therefore, this study focused on the textual meta-function so as to identify how students organize their message in texts creation. Following this aim of the study, three objectives were drawn. The first objective was to examine difficulty in thematic progression as espoused by Dane (cohesion and coherence). Secondly, the study sought to determine Ghanaian students' difficulties in thematic progression. Thirdly, the study investigated Ghanaian students' challenges in sentence construction.

With respect to the problems of thematic progression, which is the situation whereby the theme of the clause may pick up or repeat a meaning from a preceding theme or rheme to help achieve cohesion or unity and coherence in a text, by helping readers in a logical and rational course (Paltridge cited in Arunsirot, 2013), it was identified that the students have a difficulty as they introduce brand new theme in their thematic progression, resulting in disunity of sentences in their paragraph. Also, their theme development showed a misplaced theme which explains their illogical sentence constructions and incoherent ideas. Further analysis revealed that Ghanaian students struggle with proper sentence construction. In view of this, their sentences are in fragment, run on, phrases rather than sentences. It can therefore be said that ESL students lack logical and the necessary skills in presenting their ideas about a topic in the academic context. It is likely they have not been able to internalize certain structural rules and that they may not be able to effectively communicate their ideas. This could be attributed to their lack of extensive reading and also few opportunities to practice writing. Giving the challenges Ghanaian students have with paragraph development and sentence construction, it becomes essential for teachers to give students time to practice writing. Teachers must take time to read and guide students regarding proper structuring of sentences and logical sequence of ideas. For teachers, especially teachers of English language to be able to engage students in the practice of writing, student - teacher ratio must be quite appreciable. This study considered specifically the quality of ESL students' writing skills, future studies can consider the pervasive nature of the writing problems of ESL students.

\section{References}

Adas, D. \& Bakir, A. (2013). Writing difficulties and new solutions: Blended learning as an approach to improve 
writing abilities. International Journal of Humanities and Social Science, 3(9), 254-256.

Adika, K.S. (2012) English in Ghana: Growth, tensions, and trends. International Journal of Language, Translation and Intercultural Communication. 1(1), 151-166.

Abdulkareem, M. N. (2014). An investigative study of academic writing problems faced by Arab postgraduate students at University Technology Malaysia (UTM). Theory and Practice in Language Studies,3(9), 15521557.

Afful, J. B. A. (2007). Academic literacy and communication skills in the Ghanaian university: A proposal. Nebula, 4(3), 141-159.

Alfaki, I. M. (2015). University students' English writing problems: Diagnosis and remedy. International Journal of English Language Teaching, 3(3), 40-52.

Al Seyabi, F. \& Tuzlukova, V. (2014). Writing problems and strategies: An investigative study in the Omani school and university contexts. Asian Journal of Social Sciences \& Humanities, 3(4), 37-48.

Amua-Sekyi, E.T. (2010). The status of English as a subject in an English medium context: the Ghanaian situation. The Oguaa Educator, 1, 18-27.

Arancon, R. P. (2013). The use of SFL genre theory for the analysis of students' writing skills in ESP. Volumen Monográfico, 7, 245-262.

Arunsirot, S. (2013). An analysis of textual metafunction in Thai EFL students' writing. Research on Youth and Language, 7(2), 160-174.

Danes, F. (1974). Functional sentence perspective and the organization of the text. Papers on Functional Sentence Perspective, 23, 106-128.

Ghabool, N., Mariadass, M. E. \& Kashef, S. H. (2012). Investigating Malaysian ESL students' writing problems on conventions, punctuation, and language use at secondary school level. Journal of Studies in Education, 2(3), 130-143.

Klu, E. K. (2014). An analysis of grammatical concord in selected examination scripts of students of the Ghana Technology University College. International Journal of Education Science, 7(3), 727-731.

Krishnamurthy, S., Kangira, J., Tjiramanga, A. \& Beukes, B. (2010). An analysis of English errors made by Polytechnic of Namibia students. Journal of Language and Communication, 4(5), 1-15.

Lawoyi, A. A. \& Adeyanju, A. (2013). Errors in the written English of students in two central Nigeria Polytechnics: An assessment. Journal of ELT and Poetry, 1(1), 16-39.

Msanjila, Y. P. (2005). Problems of writing in Kiswahili: A case study of Kigurunyembe and Morogoro Secondary Schools in Tanzania. Nordic Journal of African Studies, 14(1), 15-25.

Negari, G. M. (2011). A study on strategy instruction and EFL learners' writing skill. International Journal of English Linguistics, 1(2), 299-307.

Ogah (2013). Decision making in the research process: Companion to students and beginning researchers. Legon, Accra: Adwinsa Publications Ltd.

Omotese, F. E. (2012). A comparison of errors of concord students often conflict. Elixir International Journal, 49, 9862-9864

Sekyi-Baidoo, Y. (2003). Learning and communicating ( $2^{\text {nd }}$ ed). Accra: Infinity Graphics Ltd.

Sey, K.A. (1973). Ghanaian English. London: Macmillan.

Snape, D. \& Spencer L. (2003). The foundations of qualitative research. London: Sage Publication Ltd. 